\title{
SAR IMAGE DE-NOISING BASED ON SHEAR-DUAL TREE COMPLEX WAVELET TRANSFORM
}

\author{
Shuaiqi Liu ${ }^{a, b}$, Yu Zhanga,b, Xiaole Maa, Ming Liuc, \\ Shaohai $\mathrm{Hu}^{\mathrm{d}}$ and Jie Zhao ${ }^{\mathrm{a}, \mathrm{b}}$ \\ ${ }^{a}$ College of Electronic and Information Engineering, Hebei University, Baoding \\ Hebei 071002, P. R. China \\ ${ }^{b}$ Key Laboratary of Digital Medical Engineering of Hebei Province Baoding Hebei \\ 071002, P. R. China \\ 'Personnel Division, Hebei University, Baoding Hebei 071002, P. R. China \\ dInstitute of Information Science, Beijing Jiaotong University, Beijing 100044, \\ P. R. China
}

\begin{abstract}
\footnotetext{
${ }^{*}$ Corresponding author.

E-mail address: shdkj-1918@163.com (Shuaiqi Liu).
}

Copyright ( 2016 Scientific Advances Publishers

2010 Mathematics Subject Classification: 80.

Submitted by Bin Guo.

Received March 12, 2016
\end{abstract}

Speckle suppression is a hot research field in the world. In this paper, combining the advantages of shear filtering and dual tree complex wavelet transform (DTCWT), we propose a new SAR image de-noising algorithm based on K-singular value decomposition (KSVD) and soft thresholding. The new algorithm uses shear filtering to do directional decomposition. After that, DTCWT is applied to do scale decomposition. Soft thresholding is applied to high frequency coefficients, while K-SVD is applied to low frequency coefficients. The new algorithm can not only remove most noise in the high frequency coefficients, but 
also remove a small amount of noise contained in the low frequency. The experimental results show that our method can both improve peak signal to noise ratio (PSNR) and enhance the image visual effect.

Keywords: SAR de-noising, shearlet, DTCWT, K-SVD.

\section{Introduction}

Recently, with the constant improvement and development of wavelet transform theory, it is more widely applied in the field of image processing. Moreover, many scholars apply it to the SAR image denoising [1]. But, two-dimensional wavelet only has limited direction and cannot be better to express two-dimensional images with line or surface $[2,3]$. To overcome these disadvantages, many scholars put forward all kinds of multi-scale geometric transform, and the popular multi-scale geometric transforms used in speckle suppression are dual tree complex wavelet transform (DTCWT), contourlet and curvelet [2-9]. However, DTCWT is not an optimal sparse representation transform. And contourlet is not shift invariance. What is more, curvelet is not constructed by single basis function, which leads to not conforming to the multi-resolution framework and many problems in the discretization [5]. In order to overcome the shortcomings of the transform above, according to the theory of tight frame, shearlet transform is proposed based on strict mathematical logic reasoning. Its approximation order of the image is as same as curvelet, but its implementation is more simple and flexible. Therefore, as the shearlet is proposed, it causes the attention of many scholars. And it is quickly applied to image processing. With the gradual in-depth research on shearlet, it is widely used in speckle suppression. In this paper, combining the characteristics of shear filtering and dual tree complex wavelet, we propose a new transform called shear-dual tree complex wavelet transform (SDTCWT) which cascaded shear filtering before DTCWT. SDTCWT is shift invariance and also can add a number of low sub-bands, so we propose a new SAR images de-noising based on SDTCWT. 
Firstly, SDTCWT is applied to SAR image, and we can get the high frequency and low frequency of the image. Secondly, the noise of the high frequency is de-noised by soft thresholding, while the noise of the low frequency is de-noised by $\mathrm{K}$-singular value decomposition (K-SVD) algorithm. Lastly, the de-noising image is obtained by inverse SDTCWT with Lee filtering. Compared with other de-noising algorithms, our method effectively improves the peak signal to noise ratio (PSNR) of de-noising image and the image visual effect. What is more, de-noising image is smoother and it improves the computing speed significantly.

\section{Shear-Dual Tree Complex Wavelet Transform (SDTCWT)}

Shearlet lacks shift invariance because Laplacian pyramid is used to do scale decompostion. If replacing the Laplacian pyramid with the downsampling Laplacian pyramid, the redundancy of shearlet is greatly increasing (in terms of decomposition scale $J$, the redundancy is $2^{J}$ ), which results in the decrease of computation speed greatly.

DTCWT just needs limited redundancy to realize shift invariance. What is more, when the decomposition scale increases, the redundancy always is 4 . So the speed of the algorithm has been effectively improved. The same to [9], combining the advantages of DTCWT and shear filtering, this paper proposes a new transform called SDTCWT. Similar to the architectural approaches of the NSDFB-DTCWT [9], we cascade shear filtering before DTCWT to construct the SDTCWT. SDTCWT can be described by following steps:

Step 1. Direction sub-bands can be got by applying shear filtering to image.

Step 2. Low and high sub-bands can be obtained by applying DTCWT to each direction sub-band.

Step 3. Then we can get the pyramid by repeating above operation to the low frequency sub-bands like DWT.

The reconstruction algorithm of NSDFB-DTCWT is in reverse order. 


\section{Image De-noising Algorithm}

\subsection{K-singular value decomposition}

The de-noising methods based on sparse representation are one of the mainstream methods in image de-noising. K-SVD algorithm is a popular de-noising method [10]. In this algorithm, cosine dictionary, multi-scale ridgelet dictionary [11], Gabor dictionary [12] and so on are considered to be the initial dictionary and then use the orthogonal matching pursuit (OMP) algorithm to process the images. In K-SVD, sparse decomposition and dictionary update jointly together to de-noise image with Gaussian noise.

If the dictionary $D$ is constant, and the noisy image is decomposed sparsely by dictionary $D$. Then, the de-noising model of K-SVD can be represented by follows equation:

$$
\min _{\alpha}\|\alpha\|_{0}, \text { s.t }\|I-D \alpha\|_{2} \leq \varepsilon,
$$

where \|\|$_{0}$ denotes $l_{0}$ norm, and $I \in R^{n \times m}$ denotes the noise image; $D \in R^{n \times \lambda}$ denotes the dictionary and $\lambda$ is the number of atoms in it; $\alpha \in R^{\lambda \times m}$ denotes the decomposition coefficient of the dictionary; and $\varepsilon$ is maximum error. Under a certain $\varepsilon, \alpha$ can be calculated by (1).

Using $\alpha$ to update the dictionary, $\alpha$ and the dictionary $D$ update each atom in the dictionary in turn. Suppose $d_{y}$ denotes the first column vector $y$ in the update dictionary, so coefficient of the model is:

$$
\|I-D \alpha\|_{F}^{2}=\left\|\left(I-\sum_{j \neq y} d_{j} \alpha^{y}\right)-d_{y} \alpha^{y}\right\|_{F}^{2}=\left\|E_{y}-d_{y} \alpha^{y}\right\|_{F}^{2}
$$

where $\alpha^{y}$ denotes the first row vector $y$ in the coefficient matrix $\alpha$ corresponding with $d_{y}$, and $E_{y}$ is the decomposing error of image $I$ that has removed the first $y$ atom. Usually, the matrix of whose rank is 1 is used to instead of $d_{y}$.

Finally, update all the atoms in the dictionary in turn and use the decomposition coefficient $\alpha$ and the updated dictionary to reconstruct the image after de-noising processing. 


\subsection{Soft thresholding de-noising}

Donoho put forward thresholding de-noising method in 1995, including soft thresholding and hard thresholding. Although hard thresholding can better retain the image edge information, this function is not continuity. So, hard thresholding will cause some discontinuities and ringing in image de-noising. The processing of the soft thresholding method is relatively smooth. Therefore, the high sub-bands are denoising by soft thresholding in our paper.

Soft thresholding de-noising function is shown as follows:

$$
y(x)=\left\{\begin{array}{l}
\operatorname{sgn}(x)(|x|-T), \text { if }|x| \geq T, \\
0, \text { otherwise, }
\end{array}\right.
$$

where $T$ is the critical threshold, and $\operatorname{sgn}($.$) is symbolic function.$

\subsection{Lee filter}

Under minimum mean square error criterion, Lee filter estimates the nonlinear multiplicative noise signal by using local mean and variance. Suppose noise image is $I$, and image without noise is $X$, and the mean of $I$ and $X$ are $\bar{I}$ and $\bar{X}$, then $\bar{X}=E(X), \bar{I}=E(I)$. Suppose the forecast image $\hat{X}$ is the linear combination of the average image $\bar{X}$ and the observation image $I$. Then, we can get

$$
\hat{X}=a * \bar{X}+b * I,
$$

where $a$ and $b$ are the solution that makes the mean square error term be minimum. That is,

$$
a=1-b, \quad b=\frac{\delta_{x}}{\delta_{1}},
$$

where $\delta_{1}$ denotes the variance of $I$. Then,

$$
\delta_{x}=\frac{\delta_{1}-\sigma_{v} \bar{I}^{2}}{1+\sigma_{v}^{2}},
$$


where

$$
\sigma_{v}=\frac{1}{\sqrt{\bar{N}}}
$$

where $\bar{N}$ denotes number of looks. In the general situation, number of looks is known.

\section{SAR Image De-noising Based on SDTCWT}

\subsection{The experimental steps}

Usually, speckle is fully developed [13], and it is multiplicative noise. It obeys the distribution of $\Gamma$ and it is second order stationarity. Besides, the mean of speckle is 1 , and the variance is inversely proportional to the equivalent number of looks (ENL). Before removing the speckle, generally, logarithm transform is applied to noise image so that the speckle can be converted to additive noise. In our paper, the steps of the proposed method are described as follows:

Step 1. Logarithm transform is applied to the noised image.

Step 2. SDTCWT is applied to noise image, and we can get low subbands and high sub-bands coefficients.

Step 3. K-SVD is applied to low sub-bands coefficients to remove the noise in low sub-bands. And soft thresholding is applied to high subbands coefficients to suppress main noise.

Step 4. Reconstruct image is gotten by SDTCWT after de-noising.

Step 5. After indexing the reconstruction image, deal it with Lee filtering and we can get the final de-noised image.

\subsection{The experimental results}

In order to verify the reliability and validity of the proposed algorithm in this paper, our method is compared with de-noising algorithm based on shearlet transform (ST) in [7], de-noising method based on shearlet transform by using the context-based model (ST-C) in $[5]$. 
Figure 1 shows the de-noised image by different method, and the original image is in the image library of Stanford University. The SAR image of fields in Figure 2 is gotten by Terra Sar-X. As can be seen in Figure 1 and Figure 2, compared with other de-noising algorithms, our algorithm has better visual effect.

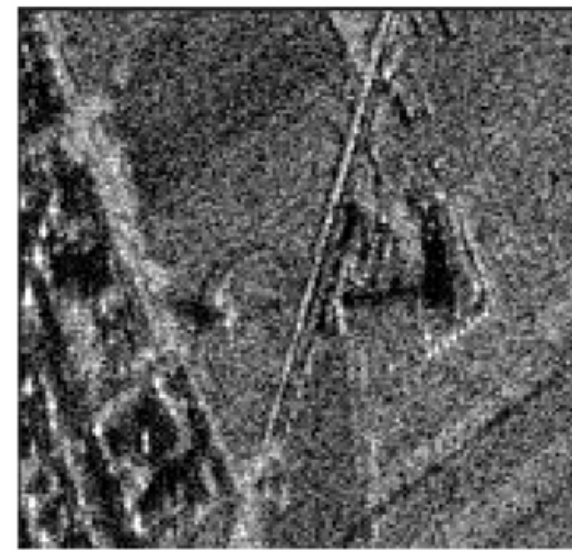

(a)

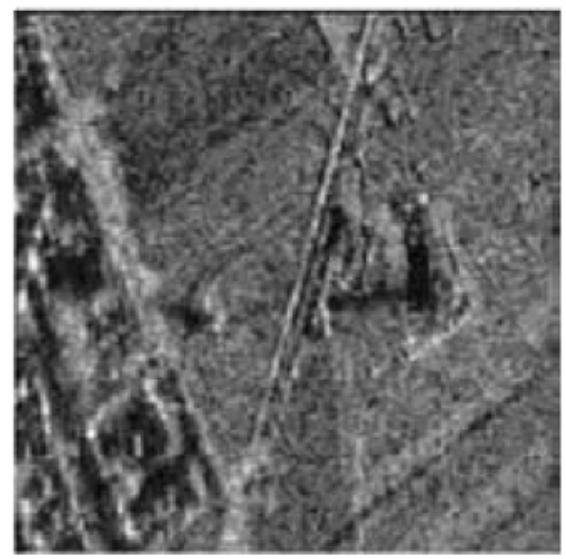

(c)

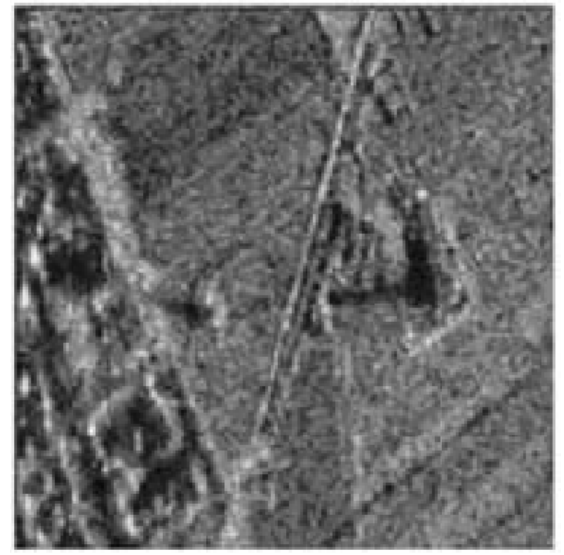

(b)

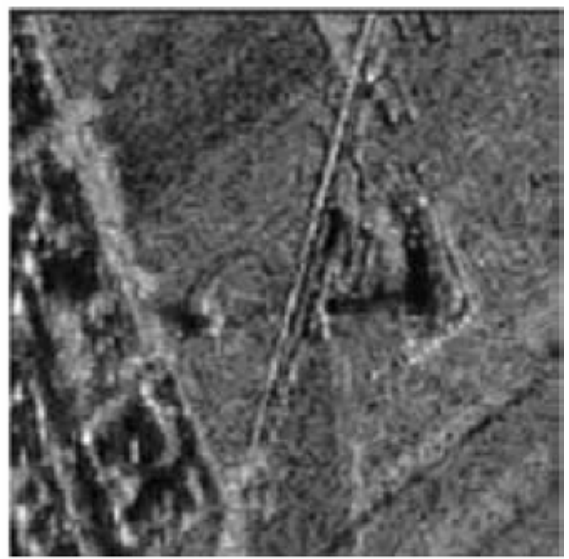

(d)

Figure 1. The results by each de-noising method. (a) The original SAR image, (b) de-noising by ST, (c) de-noising by ST-C, and (d) de-noising by the proposed. 


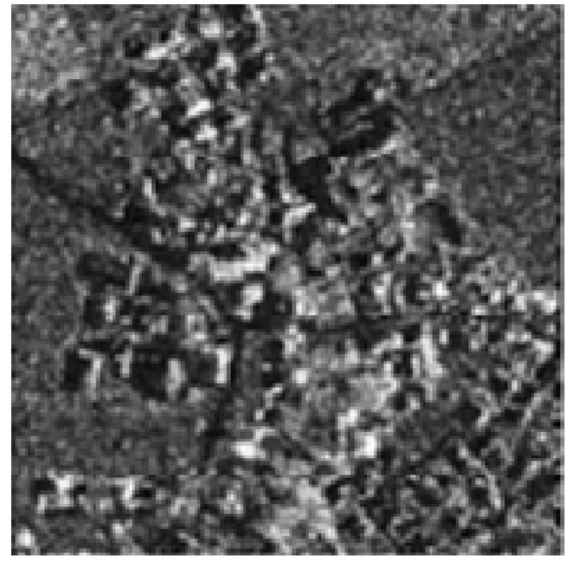

(a)

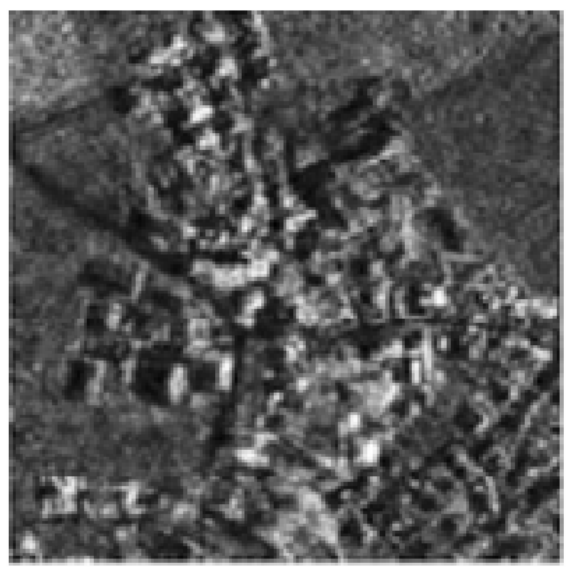

(c)

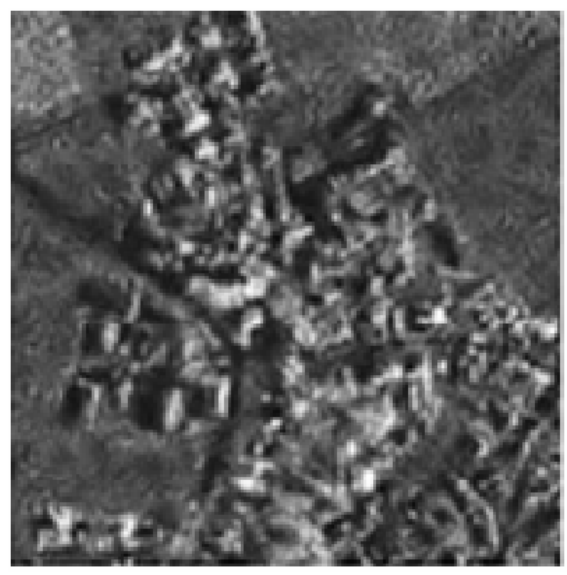

(b)

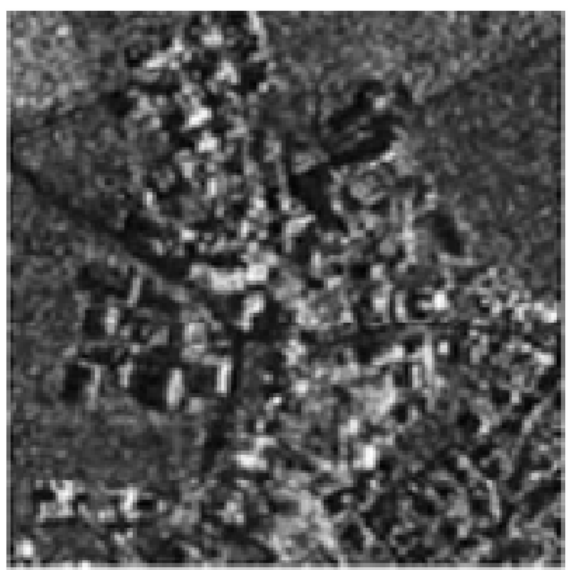

(d)

Figure 2. The results by each de-noising method for the SAR image of fields. (a) The noise image of fields, (b) de-noising by ST, (c) de-noising by ST-C, and (d) de-noising by the proposed.

In order to evaluate the performance of the algorithms above more objectively, we can calculate some common parameters. And these de-noising performance parameters include the peak signal-to-noise ratio (PSNR), equivalent number of looks (ENL), standard deviation (Sd), and edge preservation index (EPI), shown in Table 1 and Table 2. What is 
more, larger the PSNR is, stronger the capability of de-noising algorithm is; greater the ENL is, better the visual effects of the de-noising images are; and greater the EPI is, more details of the images can be preserved. From Table 1 and Table 2, our algorithm has higher PSNR. The image which is obtained by our algorithm not only has smooth image, but also suppresses artificial texture, and obviously improves the image visual effect.

Table 1. Performance parameters of Figure 1 by different de-noising methods

\begin{tabular}{|c|c|c|c|c|}
\hline $\begin{array}{c}\text { De-noising } \\
\text { method }\end{array}$ & $\begin{array}{c}\text { PSNR } \\
(\mathrm{dB})\end{array}$ & ENL & Sd & EPI \\
\hline ST & 28.15 & 13.74 & 36.41 & 0.91 \\
ST-C & 31.62 & 15.77 & 37.35 & 0.94 \\
The proposed & 32.43 & 22.16 & 38.56 & 0.96 \\
\hline
\end{tabular}

Table 2. Performance parameters of Figure 2 by different de-noising methods

\begin{tabular}{|c|c|c|c|c|}
\hline $\begin{array}{c}\text { De-noising } \\
\text { method }\end{array}$ & $\begin{array}{c}\text { PSNR } \\
(\mathrm{dB})\end{array}$ & ENL & Sd & EPI \\
\hline ST & 28.62 & 14.06 & 35.64 & 0.92 \\
ST-C & 31.87 & 15.94 & 36.53 & 0.94 \\
The proposed & 33.12 & 22.67 & 37.36 & 0.97 \\
\hline
\end{tabular}

\section{Conclusion}

This paper puts forward a new SAR image de-noising algorithm based on SDTCWT. As can be seen in the experimental results, there are some advantages of the proposed method. On one hand, the algorithm can improve the peak signal to noise ratio of the image, and speckle noise of the image gets effective suppression. On the other hand, the algorithm can not only improve the image visual effect, but also inhibit the generation of artificial texture. But, the proposed algorithm is imperfect. 
EPI can be improved in subsequent studies, especially when the edge detail and texture features of the image are rich. So, the next work will be solving the problems remaining in the paper.

\section{Acknowledgements}

Our work is supported in part by Natural Science Foundation of China under grant 61401308 and 61572063, Natural Science Foundation of Hebei Province under grant 2013210094, F2016201142 and F2016201187, Natural Social Foundation of Hebei Province under grant HB15TQ015, Science research project of Hebei Province under grant QN2016085 and ZC2016040, Natural Science Foundation of Hebei University under grant 2014-303, and National Comprehensive Ability Promotion Project of Western and Central China.

\section{References}

[1] S. Q. Liu, S. H. Hu and Y. Xiao, SAR image de-noised based on wavelet-contourlet transform with cycle spinning, Signal Processing 27(6) (2011), 837-842 (in Chinese).

[2] S. Q. Liu, S. H. Hu and Y. Xiao, Bayesian shearlet shrinkage for SAR image de-noising via sparse representation, Multidimensional Systems and Signal Processing 25(4) (2014), 683-701.

[3] B. Martí-Cardona, C. López-Martínez and J. Dolz-Ripollés, Local isotropy indicator for SAR image filtering: Application to Envisat/ASAR images of the Doñana Wetland (November 2014), IEEE Journal of Selected Topics in Applied Earth Observations and Remote Sensing PP(99) (2014), 1939-1404.

[4] S. Q. Liu, S. H. Hu and Y. Xiao, Image separation using wavelets-complex shearlets dictionary, Journal of Systems Engineering and Electronics 25(2) (2014), 314-321.

[5] S. Q. Liu, M. Z. Shi, S. H. Hu et al., Synthetic aperture radar image de-noising based on shearlet transform using the context-based model, Physical Communication 13(PartC) (2014), 221-229.

[6] G. Easley, D. Labate and W. Q. Lim, Sparse directional image representations using the discrete shearlet transform, Applied and Computational Harmonic Analysis 25(1) (2008), 25-46.

[7] S. Q. Liu, S. H. Hu and Y. Xiao, SAR image de-noising based on shearlet transform, Journal of Applied Science 30(6) (2012), 629-634 (in Chinese). 
[8] S. Q. Liu, S. H. Hu and Y. Xiao, SAR image de-noising based on complex shearlet transform domain Gaussian mixture model, Acta Aeronautica et Astronautica Sinica 34(1) (2013), 173-180 (in Chinese).

[9] S. Q. Liu, S. H. Hu and Y. Xiao, SAR image de-noise base on local hybrid filter, Journal of Systems Engineering and Electronics 34(2) (2012), 17-23 (in Chinese).

[10] M. Aharon, M. Elad and A. Bruckstein, K-SVD: An algorithm for designing over complete dictionaries for sparse representation, IEEE Transactions on Image Processing 54(11) (2006), 4311-4322.

[11] C. Z. Deng, Image denoising algorithm based on multiscale ridgelet dictionary, Computer Engineering 36(23) (2010), 207-208.

[12] J. Y. Zeng, J. Y. Gan and Y. K. Zhai, Application of Gabor dictionary and SL0 norm in disguise face recognition, Applied Mechanics and Materials 29(2) (2013), 278-280.

[13] J. W. Goodman, Some fundamental properties of speckle, Journal of the Optical Society of America 66(11) (1976), 1145-1150. 